This article has been accepted for publication in Monthly Notices of the Royal Astronomical Society: Letters (c) 2017 The Authors. Published by Oxford University Press on behalf of the Royal Astronomical Society. All rights reserved. 


\title{
Cosmological constraints from a joint analysis of cosmic growth and expansion
}

\author{
M. Moresco ${ }^{1,2 \star}$ and F. Marulli ${ }^{1,2,3 \star}$ \\ ${ }^{1}$ Dipartimento di Fisica e Astronomia, Università di Bologna, Via Gobetti 93/2, I-40129, Bologna, Italy \\ ${ }^{2}$ INAF - Osservatorio Astronomico di Bologna, Via Gobetti 93/3, I-40129 Bologna, Italy \\ ${ }^{3}$ INFN - Sezione di Bologna, Viale Berti Pichat 6/2, I-40127 Bologna, Italy
}

Accepted 2017 July 4. Received 2017 July 4; in original form 2017 May 22

\begin{abstract}
Combining measurements on the expansion history of the Universe and on the growth rate of cosmic structures is key to discriminate between alternative cosmological frameworks and to test gravity. Recently, Linder proposed a new diagram to investigate the joint evolutionary track of these two quantities. In this letter, we collect the most recent cosmic growth and expansion rate data sets to provide the state-of-the-art observational constraints on this diagram. By performing a joint statistical analysis of both probes, we test the standard $\Lambda$ cold dark matter model, confirming a mild tension between cosmic microwave background predictions from Planck mission and cosmic growth measurements at low redshift $(z<2)$. Then we test alternative models allowing the variation of one single cosmological parameter at a time. In particular, we find a larger growth index than the one predicted by general relativity $\gamma=0.65_{-0.04}^{+0.05}$. However, also a standard model with total neutrino mass of $0.26 \pm 0.10 \mathrm{eV}$ provides a similarly accurate description of the current data. By simulating an additional data set consistent with next-generation dark-energy mission forecasts, we show that growth rate constraints at $z>1$ will be crucial to discriminate between alternative models.
\end{abstract}

Key words: methods: data analysis - cosmological parameters-cosmology: observations.

\section{INTRODUCTION}

Since the discovery of the accelerated expansion of the Universe (Riess et al. 1998; Perlmutter et al. 1999), different cosmological probes have been exploited to constrain the expansion history of the Universe and the growth rate of cosmic structures therein (for a comprehensive review, see e.g. Weinberg et al. 2013). The main quantities to be measured are the Hubble parameter, $H(z)=\dot{a} / a$, that describes the background expansion, and the linear growth rate $f(z)$, defined as $f=\mathrm{d} \ln G / \mathrm{d} \ln a$, where $a$ is the scale factor and $G$ is the growth factor of the matter density contrast. Usually, the quantity that is actually constrained is $f \sigma_{8}(z)$, where $\sigma_{8}$ is the matter power spectrum normalization at $8 h^{-1} \mathrm{Mpc}$.

Typically, $H(z)$ and $f \sigma_{8}(z)$ are measured separately using different cosmic probes, whose intrinsic properties make them more sensitive to some parameters and less to others. For instance, type IA supernovae ( $\mathrm{SNe}$ ) trace luminosity distances up to $z \sim 1-1.5$, cosmic chronometers provide a direct measurement of $H(z)$ up to $z \sim 2$, redshift-space distortions constrain $f \sigma_{8}(z)$ and baryon acoustic oscillations (BAO) give information on both $H(z)$ and $f \sigma_{8}(z)$, but with less redshift coverage than the other probes. It is a common

^E-mail: michele.moresco@unibo.it (MM); federico.marulli3@unibo.it (FM) practice to combine different probes to increase the accuracy on the determination of cosmological parameters, but usually information on the growth factor and expansion are used disjointly (but see e.g. Rapetti et al. 2013). Recently, Linder (2017) proposed a new diagram exploiting these two quantities together. Specifically, it has been shown that in the $H(z) / H_{0}$ versus $f \sigma_{8}(z)$ plane different cosmologies can be more easily disentangled.

In this letter, we take advantage of the most recent measurements of both $H(z)$ and $f \sigma_{8}(z)$ to explore the approach suggested by Linder (2017) from an observational perspective. The goal of this work is to collect the most recent observational data to provide the best available constraints on the $H(z) / H_{0}-f \sigma_{8}(z)$ diagram. We used data from cosmic chronometers and redshift-space distortions to constrain possible extensions to the standard flat $\Lambda$ cold dark matter(CDM) model and provide forecasts for next-generation galaxy redshift surveys.

\section{METHODS AND DATA}

To construct the $H(z) / H_{0}-f \sigma_{8}(z)$ diagram (Linder 2017), we collect the largest homogeneous data set of $H(z)$ and $f \sigma_{8}(z)$ measurements, aimed at minimizing any possible inconsistencies between different probes.

Differently from previous analyses (e.g. Rapetti et al. 2013) that constrained the expansion history of the Universe based on 


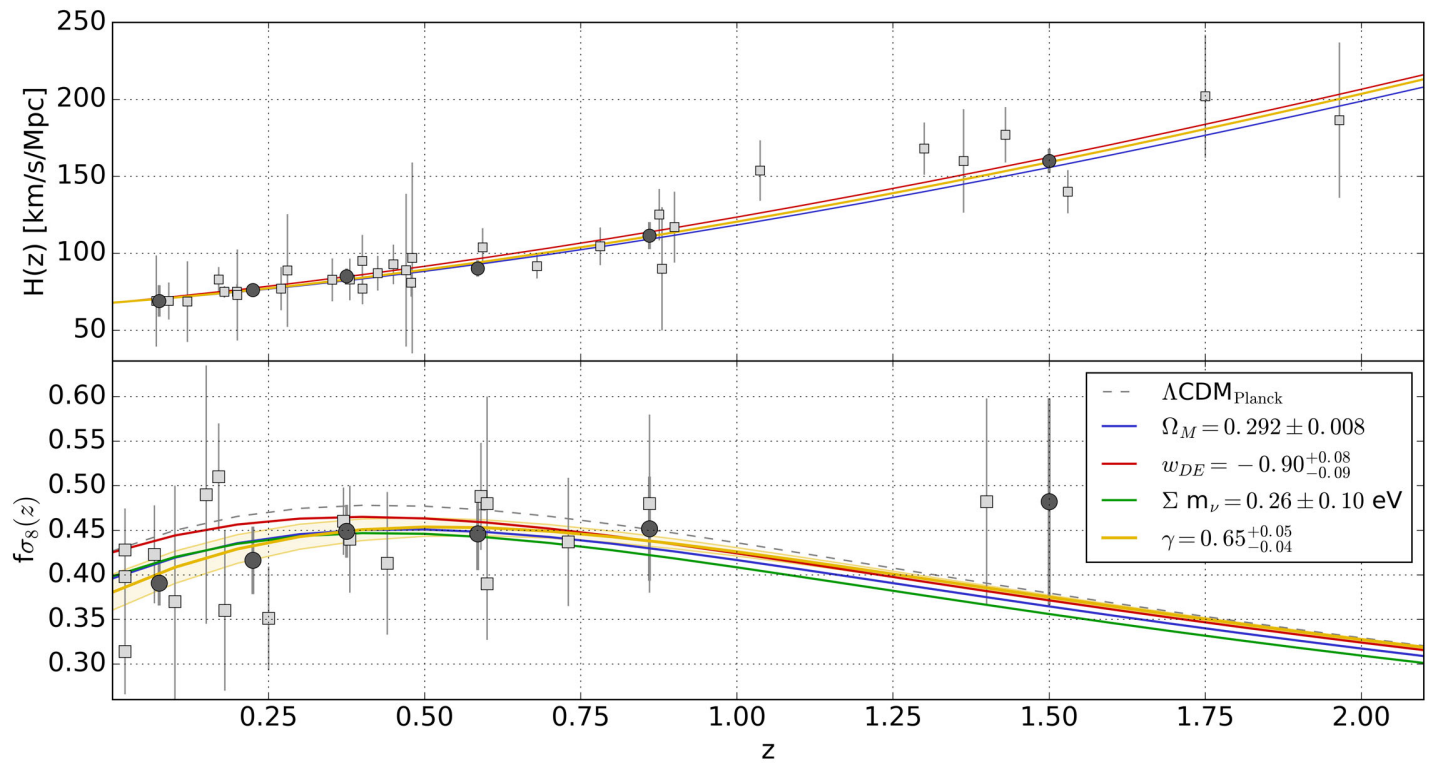

Figure 1. The redshift evolution of the Hubble parameter, $H(z)$, (upper panel), and of the linear growth rate, $f \sigma_{8}(z)$ (lower panel). The grey squares show the data used in this analysis, as described in Section 2. The black points show the binned data used to construct the $H(z) / H_{0}-f \sigma_{8}(z)$ diagram. Best-fitting models to $H(z)$ and $f \sigma_{8}(z)$ combined are shown with different lines: the dashed grey lines show the reference Planck 2016 flat $\Lambda$ CDM cosmology; while the coloured ones its extension, with free $\gamma$ (gold), $\Omega_{M}$ (blue), $\Sigma m_{v}$ (green) and $w_{\mathrm{DE}}$ (red). The yellow shaded areas show the 68 per cent confidence levels of the free $\gamma$ model for illustrative purposes.

indirect measurements, such as the luminosity distance $D_{\mathrm{L}}(z)$ from $\mathrm{SNe}$ or the acoustic-scale distance ratio $D_{\mathrm{V}}(z) / r_{\mathrm{d}}$ from $\mathrm{BAO}$, here for the first time we rely only on direct constraints on the Hubble parameter $H(z)$ obtained with the cosmic chronometer method. Originally proposed by Jimenez \& Loeb (2002), this technique has been widely tested on different galaxy-redshift surveys, providing a direct estimate of $H(z)$ without any cosmological assumption, over a large redshift range $(0<z<2$, see Moresco et al. 2016, for a detailed discussion). In this work, we use in particular the measurements provided by Simon, Verde \& Jimenez (2005), Stern et al. (2010), Moresco et al. (2012), Zhang et al. (2014), Moresco (2015), Moresco et al. (2016), Ratsimbazafy et al. (2017) that are reported in the upper panel of Fig. 1. We note that the cosmic chronometer method is quite new in the panorama of cosmological probes, and hence, while promising, it has not had the time yet to be studied to the same extent of more standard probes, such as BAO and $\mathrm{SNe}$. We refer to Weinberg et al. (2013) for a detailed review and comparison of the strengths and weaknesses of the various probes (see also Guidi, Scannapieco \& Walcher 2015; Liu et al. 2016; Goddard et al. 2017, for additional discussions).

For the linear growth rate, we consider the $f \sigma_{8}(z)$ data set recently suggested by Nesseris, Pantazis \& Perivolaropoulos (2017), which collects only the independent measurements provided by Percival et al. (2004), Davis et al. (2011), Hudson \& Turnbull (2012), Turnbull et al. (2012), Beutler et al. (2012), Samushia, Percival \& Raccanelli (2012), Blake et al. (2012), Blake et al. (2013), Feix, Nusser \& Branchini (2015), Howlett et al. (2015), Huterer et al. (2016), Chuang et al. (2016), Okumura et al. (2016), de la Torre et al. (2016). These data are shown in the lower panel of Fig. 1.

We analysed both data sets with a standard $\chi^{2}$ minimization approach. As discussed in Moresco et al. (2016) and Nesseris et al. (2017), the covariance matrix is diagonal for almost all measurements considered, except for the WiggleZ $f \sigma_{8}(z)$ data, for which we used the covariance matrix provided by Blake et al. (2012).
As reference model, we consider the baseline flat $\Lambda \mathrm{CDM}$ model obtained by Planck Collaboration et al. (2016, hereafter Planck 2016), which assumes two massless and one massive neutrino with mass $0.06 \mathrm{eV}, H_{0}=67.8 \mathrm{~km} \mathrm{~s}^{-1} \mathrm{Mpc}^{-1}, \Omega_{M}=0.308, w_{\mathrm{DE}}=-1$. We also set the value of the cosmic growth index $\gamma$ to 0.545 as predicted by general relativity, where $f(z) \simeq \Omega_{m}(z)^{\gamma}$. As already discussed in previous works (e.g. Macaulay, Wehus \& Eriksen 2013; Gil-Marín et al. 2017; Marulli et al. 2017; Nesseris et al. 2017), Planck 2016 constraints are in some tension with low-redshift measurements, in particular with $f \sigma_{8}(z)$ constraints from recent redshift-space distortion analyses. This finding is confirmed also by the present work, as can be noted in the bottom panel of Fig. 1, which shows that Planck predictions overestimate, on average, $f \sigma_{8}(z)$ measurements at $z<1$.

We explore four possible extensions to the reference $\Lambda \mathrm{CDM}$ model in order to get a better fit to the data, by changing each time one single parameter. Specifically, we vary the cosmic growth index $\gamma$, the matter density parameter $\Omega_{M}$, the total neutrino mass $\Sigma m_{v}$ and the dark-energy equation of state parameter $w_{\mathrm{DE}}$. The uncertainties in the current data are still too large to disentangle the degeneracies between the effects produced by some of these parameters, as will be shown in the following Section. Therefore, we decided to explore the effect of changing each parameter singularly.

We consider the following flat priors in the statistical analyses: $\gamma \in[0,1.5], \Omega_{M} \in[0.1,0.6], \Sigma m_{v} \in[0,1.] \mathrm{eV}$ and $w \in[-2 ., 0.2]$. We note, however, that our results are not affected by the choice of these values, since all our results are well within the considered ranges.

To investigate the sensitivity of our data to the two probes, we perform both a fit separately to $H(z)$ and $f \sigma_{8}(z)$, and a combined fit. In order to construct the $H(z) / H_{0}-f \sigma_{8}(z)$ diagram, we bin both data sets in the same redshift ranges chosen to get a uniform redshift sampling, having at least three points in both $H(z)$ and $f \sigma_{8}(z)$ bins, with the exception of the last two bins, where the sampling in $f \sigma_{8}(z)$ is very scarce. In each redshift bin, we estimate the variance weighted mean values of $H(z)$ and $f \sigma_{8}(z)$. These data are reported 


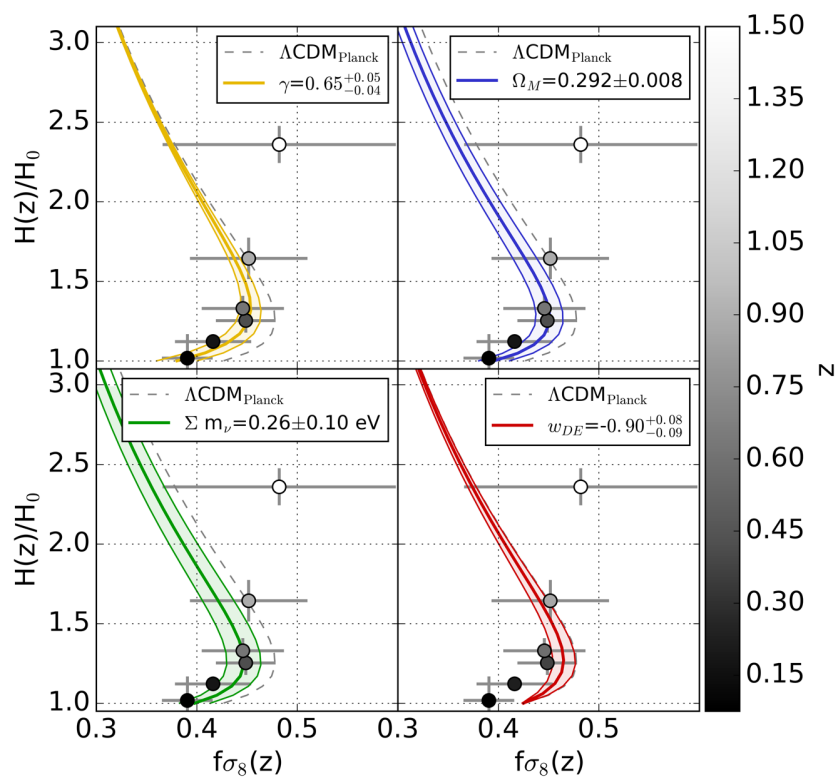

Figure 2. The $H(z) / H_{0}-f \sigma_{8}(z)$ diagram (Linder 2017): cosmic expansion versus cosmic growth. The four panels show the best-fitting models and $1 \sigma$ associated errors (coloured lines and shaded area, respectively) obtained with the different extensions to the Planck 2016 flat $\Lambda \mathrm{CDM}$ model, as indicated by the labels. The grey dashed lines show the reference Planck 2016 cosmology. The points are the observed data binned in six redshift ranges, as in Fig. 1. Their colours indicate the mean redshift of each bin, as shown by the colour bar.

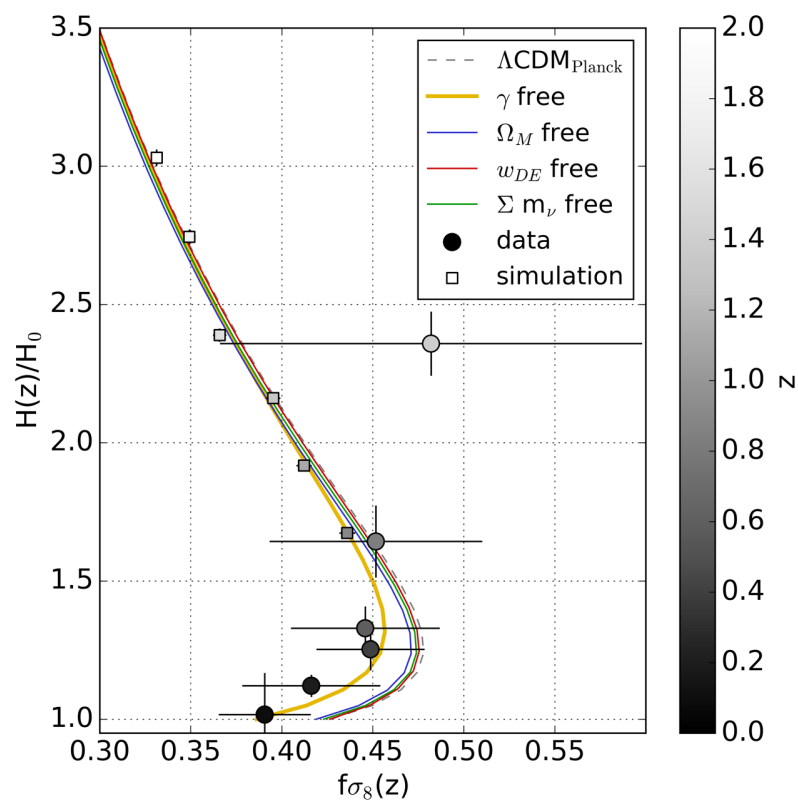

Figure 3. Same as Fig. 2, with the addition of simulated data (square points) forecasting constraints from next-generation galaxy redshift surveys. The simulated data assume a $\gamma=0.65$ extension to $\Lambda \mathrm{CDM}$. The best-fitting models are shown with the same colour code as in Fig. 2.

in Fig. 1 as a function of the mean redshift of the bins. This specific procedure is adopted purely for illustrative purposes (see Figs 2 and 3), while all statistical analyses are performed on the original unbinned data sets.

To estimate $H(z)$ and $f \sigma_{8}(z)$ in the different cosmological models considered in this work, we exploit the CosmoBolog-
naLib, a large set of Open Source C++/PYTHON libraries ${ }^{1}$ (Marulli, Veropalumbo \& Moresco 2016).

\section{ANALYSIS AND DISCUSSION}

Fig. 1 compares the best-fitting models with the $H(z)$ and $f \sigma_{8}(z)$ data sets considered in this work. The values of the best-fitting parameters of the four models considered, using $H(z)$ and $f \sigma_{8}(z)$ data both separately and combined together, are reported in Table 1 . As previously stated, these results have been obtained by allowing the variation of one single parameter at a time. The goal is to quantify how the relaxation of each cosmological parameter can reduce the tension between the reference model and the data. The current measurement uncertainties are too large to allow the variation of more than one parameter. Indeed, as we verified, the constraints are approximately a factor of 5 worse in the case of two parameters, making the analysis inconclusive.

As expected, some parameters affect only the growth of structures (i.e. $\gamma$, and to a first approximation $\Sigma m_{v}$ ), while the others have an impact also on the expansion history. In particular, all models show different evolutionary tracks that can be more clearly appreciated in the $H(z) / H_{0}-f \sigma_{8}(z)$ diagram, shown in Fig. 2. The data appear to be consistent with a higher value of $\gamma$ than the one predicted by general relativity, a lower value of $\Omega_{M}$ with respect to Planck 2016 constraints, a value of $\Sigma m_{v}$ significantly larger than the reference one, and a value of $w_{\mathrm{DE}}$ smaller than the $\Lambda$ case. By simple relaxing one single parameter, it is possible to significantly reduce the tension between the data and the reference model. Both the $\gamma, \Omega_{M}$ and $\Sigma m_{v}$ models provide an accurate description of the data, in particular at low redshift $(z<0.5)$. On the contrary, the $w_{\mathrm{DE}}$ model does not provide an appreciably better fit, converging both at low and high redshifts to the Planck 2016 reference model.

As discussed above, we decided not to include in our data sets measurements of $H(z)$ from other probes, such as from BAO (Chuang \& Wang 2012; Blake et al. 2012; Font-Ribera et al. 2014; Delubac et al. 2015), to avoid mixing systematics from different probes that may bias the results. However, we verified that our results when including these data are consistent within $1 \sigma$ with the ones obtained with the data set considered, except for $w_{\mathrm{DE}}$, which results closer to -1 and yet more at odds with lower-redshifts $f \sigma_{8}(z)$ measurements. We tested also different data sets of $f \sigma_{8}(z)$ obtained with different techniques (e.g. Hawken et al. 2017; Pezzotta et al. 2017), finding consistent results.

To test the significance of these results, we exploit two different selection model criteria, that is the Akaike Information Criterion (hereafter AIC Akaike 1974) and the Bayesian Information Criterion (hereafter BIC Schwarz 1978). For the first criterion, we use the updated definition by Sugiura (1978), which includes a correction when $N$ is small (hereafter $A I C_{\mathrm{c}}$ ). These methods compare the best-fitting likelihood functions of different models by weighting them by the number of free model parameters, thus penalising the overfitting of the data. The two criteria are defined as follows:

$A I C_{\mathrm{c}}=-2 \ln \mathcal{L}_{\max }+2 k+\frac{2 k(k+1)}{N-k-1}$,
$B I C=-2 \ln \mathcal{L}_{\max }+k \ln N$,

\footnotetext{
${ }^{1}$ Both the numerical libraries and the data sets analysed in this work are available at the following GitHub repository: https:/github.com/ federicomarulli/CosmoBolognaLib.
} 
Table 1. The best-fitting values of the cosmological parameters let free to vary in the four models considered, using only $H(z)$ data (first column), only $f \sigma_{8}(z)$ data (second column), or using the two data sets combined together (third column). The fourth and fifth columns report, respectively, the values of $\triangle A I C_{\mathrm{c}}$ and $\triangle B I C$ between the combined probes and the reference flat $\Lambda C D M$ Planck 2016 cosmology. The sixth and the seventh columns show the values of $\triangle A I C_{\mathrm{c}}$ and $\triangle B I C$ when also the simulated data are included.

\begin{tabular}{lcccccc}
\hline & $H(z)$ & $f \sigma_{8}(z)$ & Combined & $\begin{array}{c}\Delta A I C_{\mathrm{c}} \\
\text { (combined versus Planck 2016) }\end{array}$ & $\begin{array}{c}\Delta A I C_{\mathrm{c}} \\
\text { (combined+simulated versus Planck 2016) }\end{array}$ \\
\hline$\gamma$ free & - & $0.65_{-0.04}^{+0.05}$ & $0.65_{-0.04}^{+0.05}$ & 3.1 & 1.2 & 14.6 \\
$\Omega_{M}$ free & $0.33 \pm 0.03$ & $0.289 \pm 0.008$ & $0.292 \pm 0.008$ & 1.6 & -0.3 & 2.0 \\
$\Sigma m_{v}$ free & - & $0.26 \pm 0.10$ & $0.26 \pm 0.10$ & 2.1 & 0.2 & 3.7 \\
$w_{\text {DE }}$ free & $-0.96_{-0.12}^{+0.11}$ & $-0.79_{-0.15}^{+0.14}$ & $-0.90_{-0.09}^{+0.08}$ & -0.5 & -2.4 & 4.3 \\
\hline
\end{tabular}

where $\mathcal{L}_{\max }$ is the maximum likelihood, $k$ is the number of the degrees of freedom of each model ( $k=1$ in our cases) and $N$ is the number of data points. The BIC is the most conservative criterion between the two, disfavouring even more an increased number of free parameters. The differences between $A I C_{\mathrm{c}}$ or $B I C$ values are then used to compare the models. Specifically, a model is considered to better represent the data on the base of the Jeffrey's scale (Jeffreys 1961). According to this scale, a difference smaller than 1 is inconclusive, between 1 and 2.5 is moderate, between 2.5 and 5 is strong and greater than 5 is highly significant. Compared to the reference model, we find that the data prefer a different value of $\gamma$ and $\Sigma m_{v}$ with moderate to high significance (depending on the considered criterion) and of $\Omega_{M}$ with weak significance. On the other hand, the improvement that can be obtained with a different value of $w_{\mathrm{DE}}$ turns out to be not significant. Indeed both the $A I C_{\mathrm{c}}$ and $B I C$ indicate that the added $w_{0}$ parameter does not improve the fit in a statistically significant way with respect to the reference one.

To summarize, the data considered in this work suggest some deviations with respect to the flat $\Lambda \mathrm{CDM}$ model with one massive neutrino and Planck 2016 cosmological parameters. We get a better match to the data by assuming a larger value of $\gamma$ with respect to the one predicted by general relativity. However, the current uncertainties in the data are too large to discriminate between this model and a standard model with massive neutrinos with $\Sigma m_{v} \sim 0.26 \mathrm{eV}$. A similarly good agreement (but with a smaller confidence) can be obtained with a lower value of $\Omega_{M}$, though the required value would be in mild tension with Planck 2016 constraints. Finally, changing the value of $w_{\mathrm{DE}}$ has a marginal effect in the $H(z) / H_{0}-f \sigma_{8}(z)$ diagram.

The $H(z) / H_{0}-f \sigma_{8}(z)$ diagram appears particularly convenient to visualize the differences between alternative models, as can be appreciated in Fig. 2. In particular, it can be noted that new measurements at $z \gtrsim 1$ are required to disentangle the effects of different parameters. To quantify this finding, we simulate some additional $\left(H(z), f \sigma_{8}(\mathrm{z})\right)$ points at higher redshifts that will be provided by future dark-energy missions, such as Euclid (Laureijs et al. 2011) and Wide-Field Infrared Survey Telescope WFIRST (Spergel et al. 2013). In particular, we simulate six accurate $\left(\sigma_{\mathrm{H}(\mathrm{z})} / H(z)=\sigma_{f \sigma_{8}}(z) / f \sigma_{8}(z)=0.01\right)$ measurements in the redshift range $0.9-2$, assuming as the underlying model the $\gamma=0.65$ extension to $\Lambda \mathrm{CDM}$ that represents our best fit to the current data. The assumed uncertainties are conservative, considered e.g. the available forecasts for the Euclid mission (Amendola et al. 2016). Nevertheless, the goal of this test is just to provide rough estimates of the constraining power of next-generation galaxy redshift surveys and it is not meant to be specifically designed to provide accurate forecasts for any specific future missions.

The new simulated data are presented in Fig. 3 together with the best-fitting models discussed above. The differences with respect to the reference model are now extremely significant, as shown in Table 1. Moreover, being strongly constrained at high redshifts by these data, the models present now significant deviations at low redshifts. In particular, the additional data would allow us to distinguish the effect of $\gamma$ and $\Sigma m_{v}$ at high significance, as reported in Table 1.

\section{CONCLUSIONS}

In this letter, we exploited the largest homogeneous data set of $H(z)$ and $f \sigma_{8}(z)$ measurements currently available to construct the $H(z) / H_{0}-f \sigma_{8}(z)$ diagram, recently introduced by Linder (2017), testing the $\Lambda \mathrm{CDM}$ model and exploring possible extensions. We compared a reference flat $\Lambda \mathrm{CDM}$ model with four different extensions, each time varying a single cosmological parameter, namely $\gamma, \Omega_{M}$, $\Sigma m_{v}$ and $w_{\mathrm{DE}}$. We find that current low-redshift data appear in some tension with respect to the best-fitting model obtained from the latest cosmic microwave background analysis. Either a model with $\gamma=0.65_{-0.04}^{+0.05}$ or with $\Sigma m_{v}=0.26 \pm 0.10$ provides a better fit to the data at moderate to high statistical relevance, with respect to the reference model. Unfortunately, given the current measurement uncertainties, it is not possible to disentangle between these alternatives (Marulli et al. 2011). We thus simulated six additional $H(z)$ and $f \sigma_{8}(z)$ measurements at $z \gtrsim 1$ forecasting future dark-energy missions, such as Euclid and WFIRST, and found that these new data will allow us to distinguish between the models considered in this work with high statistical significance.

\section{ACKNOWLEDGEMENTS}

MM and FM acknowledge the grants ASI n.I/023/12/0 'Attività relative alla fase B2/C per la missione Euclid' and MIUR PRIN 20102011 'The dark Universe and the cosmic evolution of baryons: from current surveys to Euclid' and PRIN MIUR 2015 'Cosmology and Fundamental Physics: illuminating the Dark Universe with Euclid'.

\section{REFERENCES}

Akaike H., 1974, Proc. IEEE Trans. Autom. Control, 19, 716

Amendola L. et al., 2016, preprint (arXiv:1606.00180)

Beutler F. et al., 2012, MNRAS, 423, 3430

Blake C. et al., 2012, MNRAS, 425, 405

Blake C. et al., 2013, MNRAS, 436, 3089

Chuang C.-H., Wang Y., 2012, MNRAS, 426, 226

Chuang C. -H. et al., 2016, MNRAS, 461, 3781

Davis M., Nusser A., Masters K. L., Springob C., Huchra J. P., Lemson G., 2011, MNRAS, 413, 2906

de la Torre S. et al., 2016, A\&A, preprint (arXiv:1612.05647)

Delubac T. et al., 2015, A\&A, 574, A59

Feix M., Nusser A., Branchini E., 2015, Phys. Rev. Lett., 115, 011301

Font-Ribera A. et al., 2014, J. Cosmology Astropart. Phys., 5, 027 
Gil-Marín H., Percival W. J., Verde L., Brownstein J. R., Chuang C.-H., Kitaura F.-S., Rodríguez-Torres S. A., Olmstead M. D., 2017, MNRAS, 465,1757

Goddard D. et al., 2017, MNRAS, 466, 4731

Guidi G., Scannapieco C., Walcher C. J., 2015, MNRAS, 454, 2381

Hawken A. J. et al., 2017, A\&A, preprint (arXiv:1611.07046)

Howlett C., Ross A. J., Samushia L., Percival W. J., Manera M., 2015, MNRAS, 449, 848

Hudson M. J., Turnbull S. J., 2012, ApJ, 751, L30

Huterer D., Shafer D., Scolnic D., Schmidt F., 2016, J. Cosmol. Astropart. Phys., 5, 15

Jeffreys H., 1961, Theory of Probability, 3rd ed. Oxford Classic Texts in the Physical Sciences. Oxford Univ. Press, Oxford, p. 432

Jimenez R., Loeb A., 2002, ApJ, 573, 37

Laureijs R. et al., 2011, preprint (arXiv:1110.3193)

Linder E. V., 2017, Astropart. Phys., 86, 41

Liu G. C., Lu Y. J., Xie L. Z., Chen X. L., Zhao Y. H., 2016, A\&A, 585, A52

Macaulay E., Wehus I. K., Eriksen H. K., 2013, Phys. Rev. Lett., 111, 161301

Marulli F., Carbone C., Viel M., Moscardini L., Cimatti A., 2011, MNRAS, 418,346

Marulli F., Veropalumbo A., Moresco M., 2016, Astron. Comput., 14, 35

Marulli F., Veropalumbo A., Moscardini L., Cimatti A., Dolag K., 2017, A\&A, 599, A106

Moresco M., 2015, MNRAS, 450, L16

Moresco M. et al., 2012, J. Cosmology Astropart. Phys., 8, 006

Moresco M. et al., 2016, J. Cosmology Astropart. Phys., 5, 014

Nesseris S., Pantazis G., Perivolaropoulos L., 2017, Phys. Rev. D, preprint (arXiv:1703.10538)
Okumura T. et al., 2016, PASJ, 68, 38

Percival W. J. et al., 2004, MNRAS, 353, 1201

Perlmutter S. et al., 1999, ApJ, 517, 565

Pezzotta A. et al., 2017, A\&A, preprint (arXiv:1612.05645)

Planck Collaboration XIII, 2016, A\&A, 594, A13

Rapetti D., Blake C., Allen S. W., Mantz A., Parkinson D., Beutler F., 2013, MNRAS, 432, 973

Ratsimbazafy A. L., Loubser S. I., Crawford S. M., Cress C. M., Bassett

B. A., Nichol R. C., Väisänen P., 2017, MNRAS, 467, 3239

Riess A. G. et al., 1998, AJ, 116, 1009

Samushia L., Percival W. J., Raccanelli A., 2012, MNRAS, 420, 2102

Schwarz G., 1978, Ann. Stat., 6, 461

Simon J., Verde L., Jimenez R., 2005, Phys. Rev. D, 71, 123001

Spergel D. et al., 2013, preprint (arXiv:1305.5422)

Stern D., Jimenez R., Verde L., Kamionkowski M., Stanford S. A., 2010, J. Cosmology Astropart. Phys., 2, 008

Sugiura N., 1978, Commun. Stat. - Theory Methods, A7, 13

Turnbull S. J., Hudson M. J., Feldman H. A., Hicken M., Kirshner R. P., Watkins R., 2012, MNRAS, 420, 447

Weinberg D. H., Mortonson M. J., Eisenstein D. J., Hirata C., Riess A. G., Rozo E., 2013, Phys. Rep., 530, 87

Zhang C., Zhang H., Yuan S., Liu S., Zhang T.-J., Sun Y.-C., 2014, Res. Astron. Astrophys., 14, 1221

This paper has been typeset from a $\mathrm{T}_{\mathrm{E}} \mathrm{X} / \mathrm{L} \mathrm{T}_{\mathrm{E}} \mathrm{X}$ file prepared by the author. 